\title{
On an Integral due to Ramanujan, and some ideas suggested by it.
}

\author{
By Professor T. M. MacRobert.
}

(Read 1st March 1929. Received 20th September 1929).

By applying Fourier's Integral Theorem to a well-known formula, due to Cauchy, expressed in the form

$\int_{-\frac{1}{2} \pi}^{\frac{1}{2} \pi}(\cos \theta)^{\mu+\nu-2} e^{i \theta(\mu-\nu+2 \xi)} d \theta=\frac{\pi \Gamma(\mu+\nu-1)}{2^{\mu+\nu-2} \Gamma(\mu+\xi) \Gamma(\nu-\xi)},$.

where $R(\mu+\nu)>1$, Ramanujan ${ }^{1}$ has shown that

$$
\int_{-\infty}^{\infty} \frac{e^{i \hbar \xi} d \xi}{\Gamma(\mu+\xi) \Gamma(\nu-\xi)}=\left\{\begin{array}{cl}
\frac{\left(2 \cos \frac{1}{2} t\right)^{\mu+\nu-2}}{\Gamma(\mu+\nu-1)} e^{\frac{1}{2} i t(\nu-\mu)}, & |t|<\pi, \\
0 & ,|t|>\pi
\end{array}\right.
$$

where $t$ is any real number.

Since Cauchy proved his formula by contour integration, it may be of interest to show that Ramanujan's Integral can be deduced from it by the same method.

$$
\text { Let } I \equiv \frac{1}{2 \pi i} \int_{C} \frac{(2 \cos \theta)^{\mu+\nu-2} e^{i \theta(\mu-\nu)}}{\theta+\frac{1}{2} t} \frac{d \theta}{\Gamma(\mu+\nu-1)},
$$

where $C$ (Fig Ia) is a closed contour in the $\theta$-plane enclosing the point $-\frac{1}{2} t$ if $|t|<\pi$, and excluding the points $\pm \frac{1}{2} \pi:$ then

$$
I=\left\{\begin{array}{cc}
\frac{\left(2 \cos \frac{1}{2} t\right)^{\mu+\nu-2}}{\Gamma(\mu+\nu-1)} e^{\frac{1}{2} i t(\nu-\mu)}, & |t|<\pi \\
0 & ,|t|>\pi .
\end{array}\right.
$$
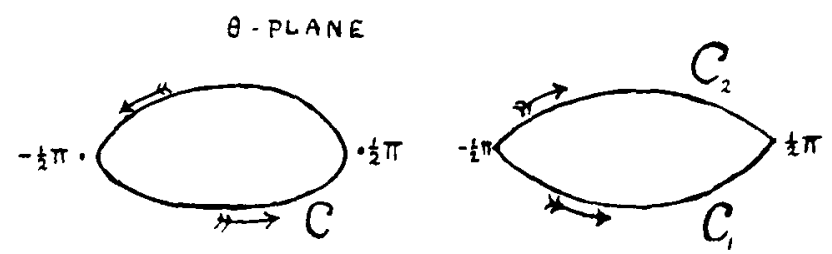

If now $R(\mu+\nu)>1$ the contour $C$ can be deformed into the contour $C_{1}$ (Fig. Ib) below the real axis from $-\frac{1}{2} \pi$ to $\frac{1}{2} \pi$ and minus

1 Quait. Joum. of Maths., 48 (1920), 294-310. 
the contour $C_{2}$ above the real axis from $-\frac{1}{2} \pi$ to $\frac{1}{2} \pi$. Then

$$
\begin{aligned}
I & =\frac{1}{\pi \Gamma(\mu+\nu-1)}\left\{\begin{array}{l}
\int_{C_{1}}(2 \cos \theta)^{\mu+\nu-2} e^{i \theta(\mu-\nu)} d \theta \int_{-\infty}^{0} e^{i \xi(\ell+2 \theta)} d \xi \\
+\int_{C_{2}}(2 \cos \theta)^{\mu+\nu-2} e^{i \theta(\mu-\nu)} d \theta \int_{0}^{\infty} e^{i \xi(t+2 \theta)} d \xi
\end{array}\right\} \\
& =\frac{1}{\pi \Gamma(\mu+\nu-1)}\left\{\begin{array}{l}
\int_{-\infty}^{0} e^{i t \xi} d \xi \int_{C_{1}}(2 \cos \theta)^{\mu+\nu-2} e^{i \theta(\mu-\nu+2 \xi)} d \theta \mid \\
+\int_{0}^{\infty} e^{i t \xi} d \xi \int_{C_{2}}(2 \cos \theta)^{\mu+\nu-2} e^{i \theta(\mu-\nu+2 \xi)} d \theta
\end{array}\right\} \\
& =\int_{-\infty}^{\infty} \frac{e^{i t \xi} d \xi}{\Gamma(\mu+\xi) \Gamma(\nu-\xi)}, \text { by (i). }
\end{aligned}
$$

It is possible, by the method indicated here, to prove many results which are usually obtained by means of Fourier's Integral Theorem. The functions involved must of course be analytic in the part of the complex plane enclosed by the contour. Two further illustrations of the procedure will now be given.

By applying Fourier's Integral Theorem to the Mehler-Dirichlet formula ${ }^{1}$

$$
P_{\zeta-\frac{1}{2}}(\cos \theta)=\frac{1}{\pi} \int_{-\theta}^{\theta} \frac{e^{i \zeta \phi} d \phi}{\sqrt{ }(2 \cos \phi-2 \cos \theta)}, 0<\theta<\pi
$$

C. Fox ${ }^{2}$ has shown that

$$
\int_{-\infty}^{\infty} P_{\zeta-\frac{1}{2}}(\cos \theta) e^{i t \zeta} d \zeta=\left\{\begin{array}{cc}
\frac{2}{\sqrt{(2 \cos t-2 \cos \theta)}}, & |t|<\theta \\
0 \quad, & |t|>\theta
\end{array}\right.
$$

where $t$ is any real number. To prove this by contour integration we make the contour $C$ (Fig. II. a) exclude the points $\pm \theta$, and then, if

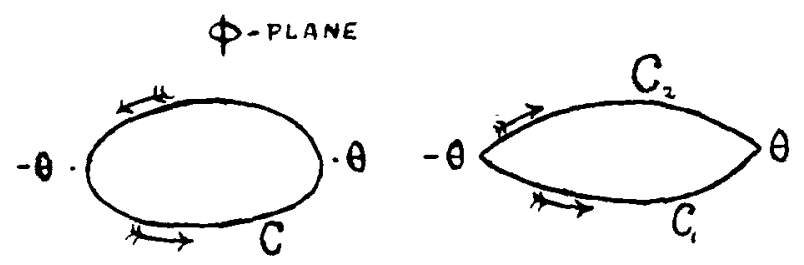

$$
\begin{aligned}
& I=\frac{1}{i \pi} \int_{C} \frac{d \phi}{(t+\phi) \sqrt{ }(2 \cos \phi-2 \cos \theta)}, \\
& I=\left\{\begin{array}{cc}
\frac{2}{\sqrt{(2 \cos t-2 \cos \theta)}}, & |t|<\theta \\
0 \quad, & |t|>\theta
\end{array}\right.
\end{aligned}
$$

J Whittaker and Watson, Modern Analysis, 2nd Ed., p. 309.

${ }^{2}$ Proc. Lond. Math. Soc., Ser. 2, 26 (1925), 76. 
Also (Fig. IIb),

$$
\begin{aligned}
I= & \frac{1}{\pi} \int_{C_{1}} \frac{d \phi}{\sqrt{ }(2 \cos \phi-2 \cos \theta)} \int_{-\infty}^{0} e^{i(t+\phi) \zeta d \zeta} \\
& +\frac{1}{\pi} \int_{C_{2}} \frac{d \phi}{\sqrt{ }(2 \cos \phi-2 \cos \theta)} \int_{0}^{\infty} e^{i(t+\phi) \zeta d \zeta}
\end{aligned}
$$

and, on changing the order of integration, and applying (iii), this gives (iv).

Similarly, from the formula ${ }^{1}$

$$
J_{n+\frac{1}{2}}(x)=e^{-\frac{1}{2} i n \pi}\left(\frac{x}{2 \pi}\right)^{\frac{1}{2}} \int_{-1}^{1} e^{i \mu x} P_{n}(\mu) d \mu
$$

which holds when $n$ is a positive integer, it can be deduced, by using contours in the $\mu$-plane between the points \pm 1 that, when $n$ is an even positive integer,

$$
e^{\frac{1}{2} i n \pi} \sqrt{ }(2 \pi) \int_{-\infty}^{\infty} e^{-i t x} J_{n+\frac{1}{2}}(x) x^{-\frac{1}{2}} d x=\left\{\begin{array}{cl}
2 \pi P_{n}(t), & |t|<1, \\
0, & |t|>1, \ldots(x)
\end{array}\right.
$$

where $t$ is any real number.

Formula for the product of two Bessel Functions of the First Kind. Ramanujan employed the integral (ii) to obtain some interesting integrals involving Bessel Functions. The form of these integrals has suggested the following formula for the product of two Bessel Functions of the first kind:

$\frac{J_{n}(x) J_{n}(y)}{x^{m} y^{n}}$

$=\frac{1}{\pi} \int_{-\frac{1}{2} \pi}^{\frac{1}{2} \pi} e^{i \theta(m-n)}\left(\frac{2 \cos \theta}{x^{2} e^{i \theta}+y^{2} e^{-i \theta}}\right)^{\frac{m+n}{2}} J_{m+n}\left[\sqrt{ }\left\{2 \cos \theta\left(x^{2} e^{i \theta}+y^{2} e^{-i \theta}\right)\right\}\right] d \theta$,

where $R(m+n)>-1$.

This can easily be verified by expanding the Bessel Function in the integrand in a power series and integrating term by term, using formula (i). If we replace $x$ and $y$ by $i x$ and $i y$ we deduce a similar formula with $I_{m}(x), I_{n}(y)$, and $I_{m+n}$ in place of $J_{m}(x), J_{n}(y)$, and $J_{m+n}$.

1 This is a special case of an integral due to Gegenbauer. For references, see Watson, Bessel Functions, p. 50. 
To illustrate the usefulness of formula (vii), it will now be applied to prove Sonine's formula ${ }^{1}$

$$
\int_{0}^{\infty} \frac{J_{m}}{t^{m-n-1}} d t=\left\{\begin{array}{cc}
\frac{r^{n}\left(1-r^{2}\right)^{m-n-1}}{2^{m-n-1} \Gamma(m-n)}, & r^{2}<1 \\
0 & , r^{2}>1
\end{array}\right.
$$

where $R(m)>R(n)>-1$.

Consider the integral

$$
I=\int_{0}^{\infty} e^{-\lambda t} J_{\nu}(a t) \frac{d t}{t^{\nu-\mu+1}}
$$

where $\lambda>0, a>0, R(\mu)>0$ : it may be written

$$
I=\frac{1}{\pi i} \int_{0}^{\infty} e^{-\lambda t}\left\{G_{\nu}(a t)-e^{i \nu \pi} G_{\nu}\left(a t e^{i \pi}\right)\right\} \frac{d t}{t^{\nu}-\mu+1} .
$$

Now break this integral into two, and, in the first, swing the path of integration through a positive right angle, in the second, through a negative right angle. This gives, if $R(\mu-2 \nu)>0$,

$$
\begin{aligned}
I= & \frac{1}{\pi i} e^{-\frac{1}{2} i \pi(\nu-\mu)} \int_{0}^{\infty} e^{-i \lambda t} G_{\nu}(i a t) \frac{d t}{t^{\nu-\mu+1}} \\
& -\frac{1}{\pi i} e^{\frac{1}{2} i \pi(\nu-\mu)+i \nu \pi} \int_{0}^{\infty} e^{i \lambda t} G_{\nu}(i a t) \frac{d t}{t^{\nu-\mu+1}} \\
= & \frac{1}{\pi i} e^{-i \pi\left(\nu-\frac{1}{2} \mu\right)} \int_{0}^{\infty} e^{-i \lambda t} K_{\nu}(a t) \frac{d t}{t^{\nu-\mu+1}} \\
& -\frac{1}{\pi i} e^{i \pi\left(\nu-\frac{1}{2} \mu\right)} \int_{0}^{\infty} e^{i \lambda t} K_{\nu}(a t) \frac{d t}{t^{\nu-\mu+1}} \\
= & \frac{2}{\pi} \int_{0}^{\infty} \sin \left\{\left(\frac{1}{2} \mu-\nu\right) \pi-\lambda t\right\} K_{\nu}(a t) \frac{d t}{t^{\nu-\mu+1}},
\end{aligned}
$$

which holds for $R(a)>0$, provided that $R(\lambda \pm i a)>0$.

Again, from (vii), if $R(m)>0, R(n)>-1, R(m-n)>0$, and

$$
\begin{aligned}
\int_{0}^{\infty} e^{-\lambda t} & \frac{J_{m}(t) J_{n}(r t)}{t^{m-n-1}} d t \\
= & \frac{r^{n}}{\pi} \int_{0}^{\infty} e^{-\lambda t} \frac{d t}{t^{m-n-1}} \int_{-\frac{1}{2} \pi}^{\frac{1}{2} \pi} e^{i \theta(m-n)}\left(\frac{2 \cos \theta}{e^{i \theta}+r^{2} e^{-i \theta}}\right)^{\frac{m+n}{2}} J_{m+n}(t \Omega) d \theta \\
= & \frac{r^{n}}{\pi} \int_{-\frac{1}{2} \pi}^{\frac{1}{2} \pi} e^{i \theta(m-n)}\left(\frac{2 \cos \theta}{e^{i \theta}+r^{2} e^{-i \theta}}\right)^{\frac{m+n}{2}} d \theta \int_{0}^{\infty} e^{-\lambda t} J_{m+n}(t \Omega) \frac{d t}{t^{m-n-1}},
\end{aligned}
$$

provided that $R(\lambda \pm i \Omega)>0$.

1 Math. Ann., $16(1880), 39$. 
Hence, from (ix), if $R(m)<1$,

$\int_{0}^{\infty} e^{-\lambda t} \frac{J_{m}(t) J_{n}(r t)}{t^{m-n-1}} d t$

where

$$
=\frac{r^{n}}{\pi} \int_{-\frac{1}{2} \pi}^{\frac{1}{2} \pi} e^{i \theta(m-n)}\left(\frac{2 \cos \theta}{e^{i \theta}+r^{2} e^{-i \theta}}\right)^{\frac{m+n}{2}} E(\theta) d \theta
$$

$$
E(\theta) \equiv \frac{2}{\pi} \int_{0}^{\infty} \sin \{(1-m) \pi-\lambda t\} K_{m+n}(t \Omega) \frac{d t}{t^{m-n-1}},
$$

which holds if $\lambda \geqslant 0$, since $|\operatorname{amp} \Omega| \leqslant \frac{1}{4} \pi$.

Now let $\lambda=0$; then, ${ }^{1}$ if $R(m)<1$,

$$
\begin{aligned}
E(\theta) & =\frac{2}{\pi} \sin (m \pi) \int_{0}^{\infty} K_{m+n}(t \Omega) \frac{d t}{t^{m-n-1}} \\
& =\frac{\Gamma(n+1)}{2^{m-n-1} \Gamma(m)} \Omega^{m-n-2}
\end{aligned}
$$

so that if $I$ is the integral on the left hand side of (viii),

$$
I=\frac{r^{n}}{\pi} \frac{\Gamma(n+1)}{2^{m-n-1} \Gamma(m)} \int_{-\frac{1}{2} \pi}^{\frac{1}{2} \pi} e^{i \theta(n-n)}(2 \cos \theta)^{m-1}\left(e^{i \theta}+r^{2} e^{-i \theta}\right)^{-n-1} d \theta,
$$

where $R(m)>0, R(n)>-1, R(m-n)>0$. The restriction $R(m)<1$ may now be removed, as the functions on both sides of the equation are holomorphic in $m$.

We next replace $\theta$ by $\frac{1}{2} \phi$, and get

$$
\begin{aligned}
I & =\frac{r^{n}}{\pi} \frac{\Gamma(n+1)}{2^{m-n} \Gamma(m)} \int_{-\pi}^{\pi} e^{\frac{1}{2} i \phi(m-n)}\left(e^{\frac{1}{2} i \phi}+e^{-\frac{1}{2} i \phi}\right)^{m-1}\left(e^{\frac{1}{2} i \phi}+r^{2} e^{-\frac{1}{2} i \phi}\right)^{-n-1} d \phi \\
& =\frac{r^{n}}{\pi} \frac{\Gamma(n+1)}{2^{m-n} \Gamma(m)} \int_{-\pi}^{\pi} e^{i \phi\left(e^{i \phi}+1\right)^{m-1}\left(e^{i \phi}+r^{2}\right)^{-n-1} d \phi} \\
& =\frac{{ }^{n}}{i \pi} \frac{\Gamma(n+1)}{2^{m-n} \Gamma(m)} \int_{C} \frac{(z+1)^{m-1}}{\left(z+r^{2}\right)^{n+1}} d z
\end{aligned}
$$

where $C$ is the unit circle described positively with the point -1 as initial point.

If $r^{2}>1$ this contour can be contracted to a point, so that $I$ has the value zero. If $r^{2}<1$, put $z=\zeta-1$, and get

$I=\frac{r^{n}}{i \pi} \frac{\Gamma(n+1)}{2^{n-n} \Gamma(m)} \int_{D} \zeta^{n-1}\left\{\zeta-\left(1-r^{2}\right)\right\}^{-n-1} d \zeta$,

where $D$ is the circle $|\zeta-1|=1$, with the origin as initial point.

\footnotetext{
1 Gray, Mathews, and MacRobert, Bessel Functions, p, 66 (11).
} 
Now let $\zeta=\left(1-r^{2}\right) z:$ then

$$
I=\frac{r^{n}}{i \pi} \frac{\Gamma(n+1)}{2^{m-n} \Gamma(m)}\left(1-r^{2}\right)^{m-n-1} \int_{\Gamma} z^{m-1}(z-1)^{-n-1} d z,
$$

where the contour $\Gamma$ starts at the origin and passes in the positive direction round the point 1 .

Hence

But the value of the integral is $2 i \sin (n+1) \pi B(m,-n)$.

from which (viii) follows.

$$
I=\frac{r^{n}\left(1-r^{2}\right)^{m-n-1}}{2^{m-n-1} \Gamma(m-n)},
$$

Note.-This method can be employed to establish Cauchy's Formula (i). For

$$
\begin{aligned}
\int_{-\frac{1}{2} \pi}^{\frac{1}{2} \pi}(\cos \theta)^{\mu+\nu-2} e^{i \theta(\mu-\nu)} d \theta \\
\quad=\frac{1}{2^{\mu+\nu-1} i} \int_{C}(z+1)^{\mu+\nu-2} z^{-\nu} d z \\
=\frac{1}{2^{\mu+\nu-1} i} \int_{D}\left(\mu+\nu-2(\zeta-1)^{-\nu} d \zeta\right. \\
=\frac{\sin \pi \nu}{2^{\mu+\nu-2}} B(\mu+\nu-1,1-\nu)=\frac{\pi \Gamma(\mu+\nu-1)}{2^{\mu+\nu-2} \Gamma(\mu) \Gamma(\nu)} .
\end{aligned}
$$

\section{ADDENDUM (added 25th November 1929)}

Formula for the product of two Hypergeometric Functions.

Formula (vii) is a simple case of the following formula for the product of two hypergeometric functions of the same kind expressed as a multiple integral of another hypergeometric function of the same kind. If, following Barnes, we employ the notation

$$
{ }_{p} F_{q}\left\{a_{r} ; \gamma_{s} ; x\right\} \equiv F\left(\alpha_{1}, a_{2}, \ldots, a_{p} ; \gamma_{1}, \gamma_{2}, \ldots, \gamma_{q} ; x\right),
$$

where $p-q \leqslant 1$, then

$$
\begin{aligned}
& \prod_{r=1}^{p} \frac{\Gamma\left(\alpha_{r}\right) \Gamma\left(\beta_{r}\right)}{\Gamma\left(\alpha_{r}+\beta_{r}\right)} \prod_{s=1}^{q} \frac{\Gamma\left(\gamma_{s}+\delta_{s}-1\right)}{\Gamma\left(\gamma_{s}\right) \Gamma\left(\delta_{s}\right)} \\
& { }_{1} F_{q}\left\{\alpha_{r} ; \gamma_{s} ; x\right\}{ }_{p} F_{q}\left\{\beta_{r} ; \delta_{s} ; y\right\} \\
& =\int_{0}^{1} \lambda_{1} \alpha_{1}-1\left(1-\lambda_{1}\right)^{\beta_{1}-1} d \lambda_{1} \int_{0}^{1} \lambda_{2} a_{2}-1\left(1-\lambda_{2}\right)^{\beta_{2}-1} d \lambda_{2} \ldots
\end{aligned}
$$

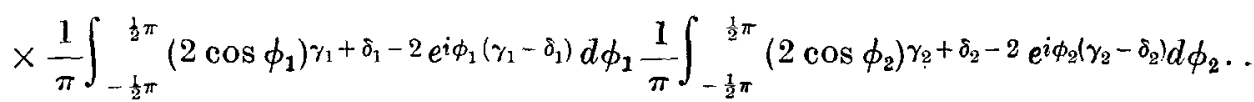

$$
\begin{aligned}
& \times{ }_{p} F_{q}\left(\alpha_{r}+\beta_{r} ; \gamma_{s}+\delta_{s}-1 ; z\right),
\end{aligned}
$$


where

$z=\prod_{s=1}^{q}\left(2 \cos \phi_{s}\right) \times\left\{\prod_{r=1}^{p}\left(\lambda_{r}\right) e^{i \Sigma \phi_{s} x}+\prod_{r=1}^{p}\left(1-\lambda_{r}\right) e^{-i \Sigma \phi_{s}} y\right\}$

and $R\left(\alpha_{r}\right)>0, \quad R\left(\beta_{r}\right)>0, \quad r=1,2, \ldots, p$,

$R\left(\gamma_{s}+\delta_{s}\right)>1, s=1,2, \ldots, q$,

one integral of the first type corresponding to each $\alpha$, and one of the second type to each $\gamma$. When $p=0$ and $q=1$ the hypergeometric function can be expressed in terms of a Bessel Function, and the formula reduces to (vii). When $p=1$ and $q=0$ the hypergeometric function becomes a binomial expression, and the formula reduces to the known formula

$$
\begin{aligned}
B(m, n)(1+x)^{-m}(1+y)^{-n} & \\
& =\int_{0}^{1} \lambda^{m-1}(1-\lambda)^{n-1}\{1+\lambda x+(1-\lambda) y\}^{-m-n} d \lambda, \ldots \text { (xi) }
\end{aligned}
$$

where $|x|<1,|y|<1, R(m)>0, R(n)>0$. 